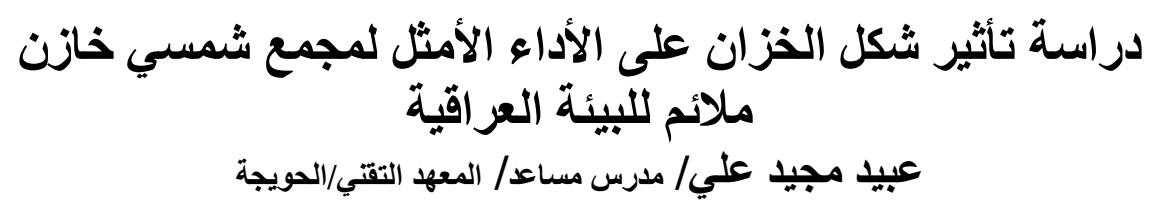

الخلاصة

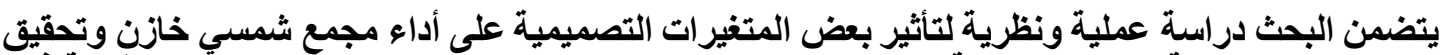

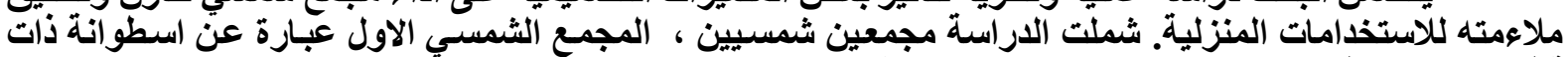

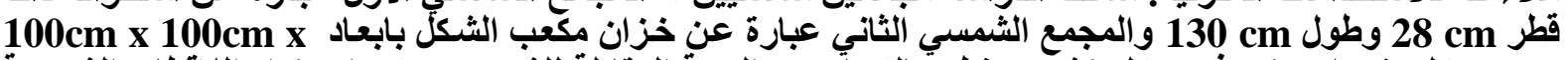

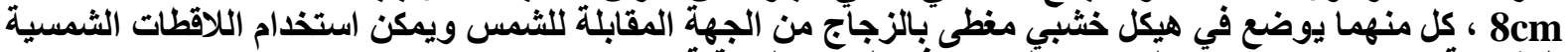

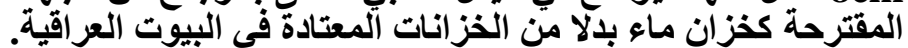

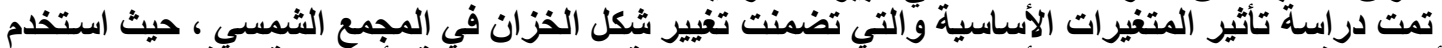

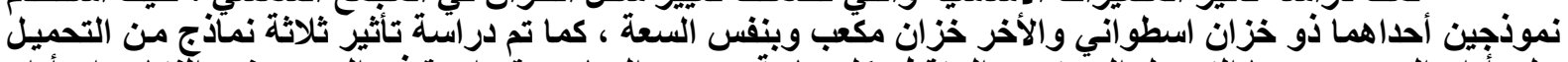

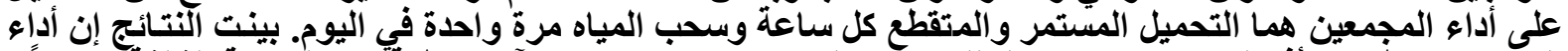

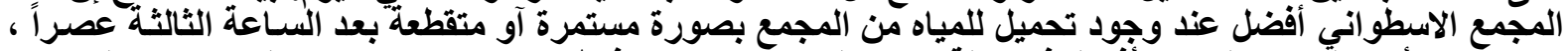

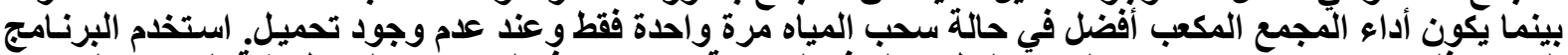

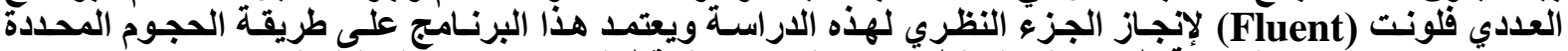

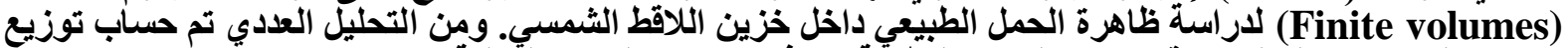

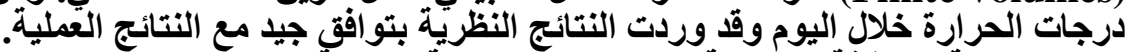

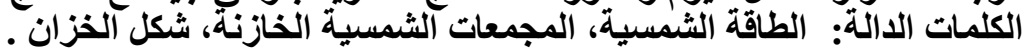

\title{
Effect of Storage Heater Shape on Performance of Built -In - Storage Solar Collector Suitable for IRAQ Environment
}

\author{
Obed Majeed Ali / Asst. Lecturer /Technical Institute of Haweja
}

\begin{abstract}
This research includes a theoretical and experimental study to the effect of some designing parameters on the performance of storage solar collector and to verify its suitability in the domestic uses. Two solar collectors studies, the first collector is cylindrical with $28 \mathrm{~cm}$ diameter and 130 height, and the second collector is cubic with dimensions $100 \mathrm{~cm} \times 100 \mathrm{~cm} \times 8 \mathrm{~cm}$, each one putting in a woody frame. Ordinary window glass was used as the top transparent cover for surface facing the sun. This storage collectors can be used as storage water tank to replace the ordinary tanks commonly used in Iraqi houses.

The basic parameters were investigated systematically, these included the storage tank shape of solar collector, two models were taken, the first, cylindrical tank storage collector, and the second cubical tank storage collector having equal capacity. The other parameters was studied, the effect of loading. Loading on the storage collector were carried out with three different water withdrawal patterns, continuous loading, an intermittent loading, all water withdrawal one time in the day. The results indicate that the performance of cylindrical collector was better than the cubic collector in the continuous loading and intermittent loading after 3 PM, and the performance of cubic collector was better than cylindrical collector when all water withdrawal in the day at no load conditions.

To verify the experimental test results, a numerical study was carried out using Fluent program. The natural convection phenomenon in the enclosure of the storage collector was investigated based on the method of control volume. From the Fluent results and analysis of the problem, the temperature distribution throughout the day was obtained. The Fluent results agreed well with the experimental data obtained.
\end{abstract}

Key wards: Solar Energy ,Storage Solar Collectors, Storage Tank Shape •

$$
\text { قبل: } 14 \text { - } 6011 \text { - }
$$$$
\text { أستلم: } 4 \text { - 2010 - 2010 - 20 }
$$ 


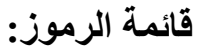

\begin{tabular}{|c|c|c|}
\hline الوحدات & 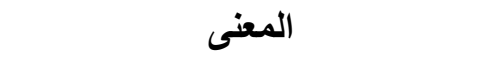 & 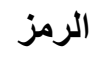 \\
\hline $\mathrm{m} / \mathrm{sec}$ & السرعة باتجاه X & $\bar{U}$ \\
\hline $\mathrm{m} / \mathrm{sec}$ & السر عة باتجاه y & $\mathrm{V}$ \\
\hline $\mathrm{m} / \mathrm{sec}$ & السر عة باتجاه z & $\mathrm{W}$ \\
\hline $\mathrm{kg}$ & الكتلة & $\mathrm{m}$ \\
\hline $\mathrm{kg}$ & كتلة الماء في المجمع & $\mathrm{m}_{\text {total }}$ \\
\hline${ }^{\circ} \mathrm{C}$ & درجة الحرارة & $\mathrm{T}$ \\
\hline${ }^{\circ} \mathrm{C}$ & معدل درجة حرارة المجمع الخازن & $\mathrm{T}_{\mathrm{av}}$ \\
\hline${ }^{\circ} \mathrm{C}$ & درجة حر ار الماء الخارج من الخزان & $\mathrm{T}_{\text {outlet }}$ \\
\hline- & الخاصية & $\phi$ \\
\hline $1 / \mathrm{K}$ & معامل التمدد الحجمي & $\beta$ \\
\hline $\mathrm{Kg} / \mathrm{m}^{3}$ & الكثافة الكتلية & $\rho$ \\
\hline & مرجعية & $\mathrm{O}$ \\
\hline- & الثريحة & I \\
\hline
\end{tabular}

1. - 1 المقدمة ومراجعة المصادر:

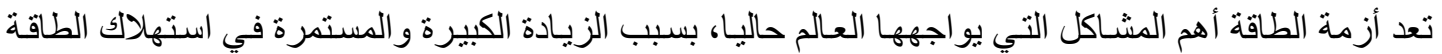

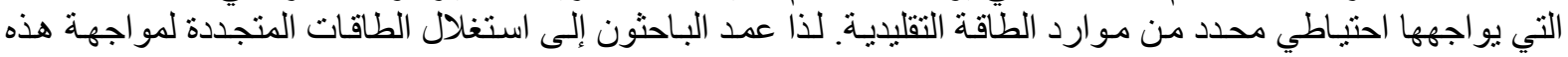

النقص الكبير في الطاقة، وكانت الطاقة الثمسية إحدى الطاقات المرشحة لاحتلال مكان الصدارة الطدان محل الطاقات التقليدية.

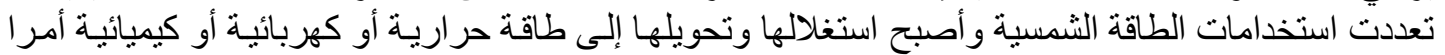

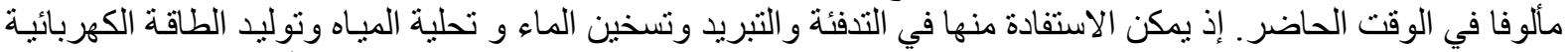

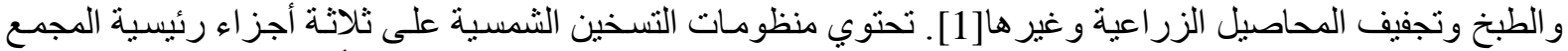

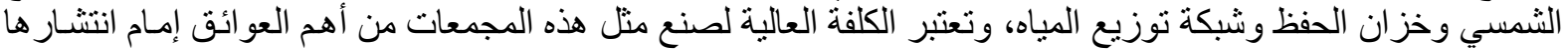

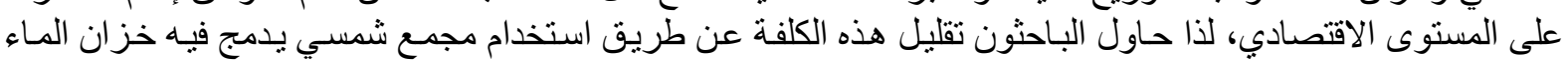

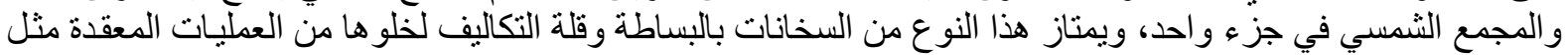

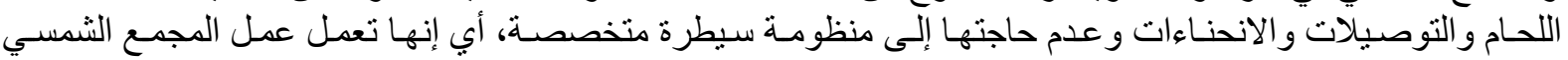

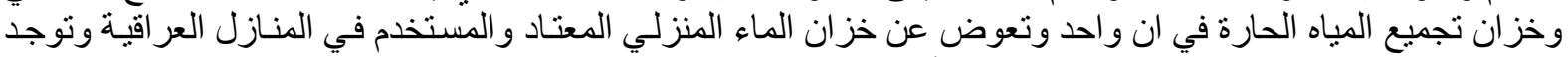

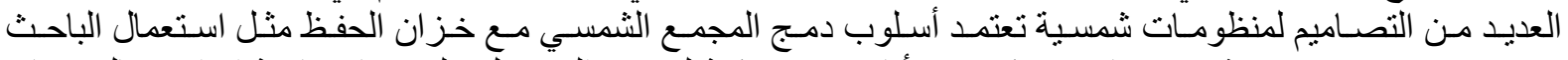

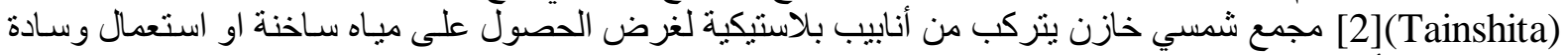

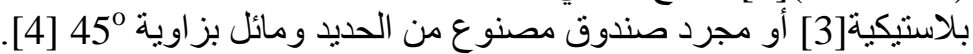

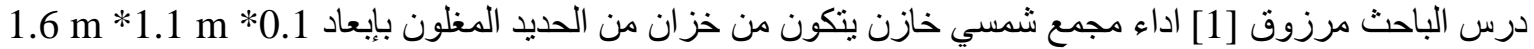

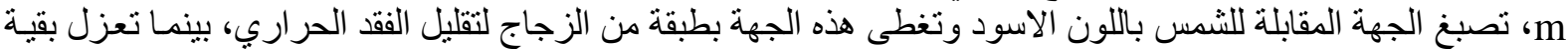

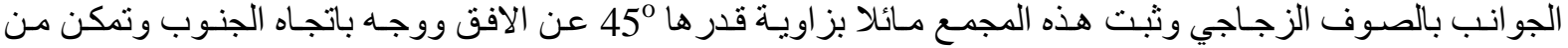

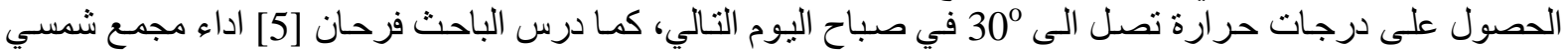

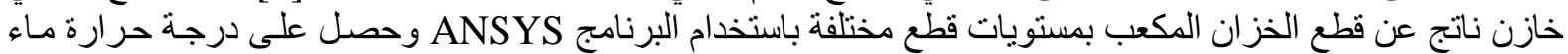

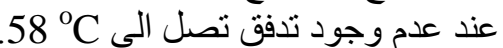

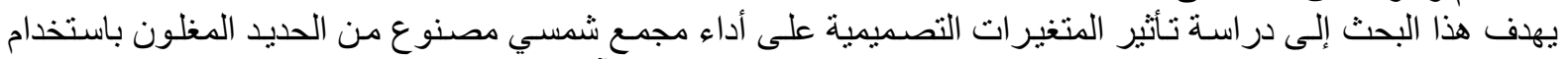
خزان على شكل اسطو انة وخز ان على شكل مكعب وبنفس السعة وتحديد الآكثر ملائمه للبيئة العر اقية.

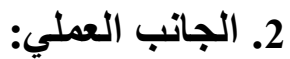

لأدر استة تأثنير المتغير ات التصميمية تم بناء نموذجين للمجمع الثمسي الخـازن، النموذج الاول يتكون من خزان

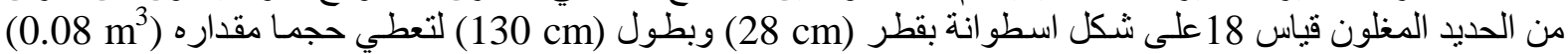

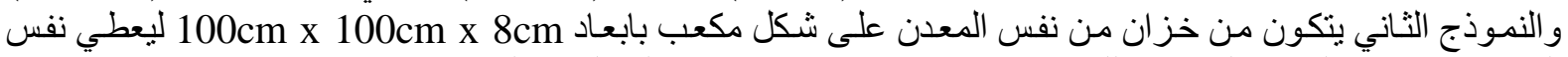

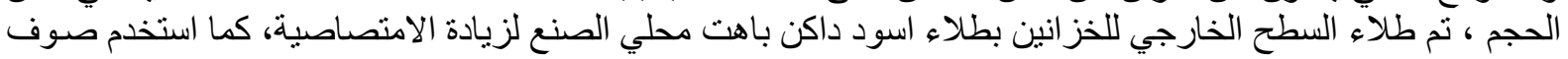




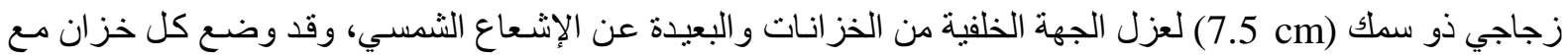

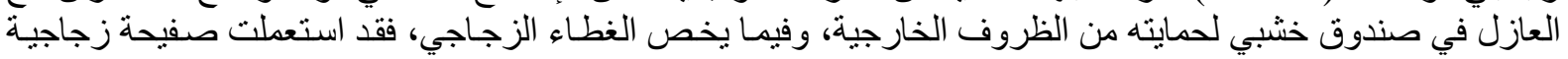

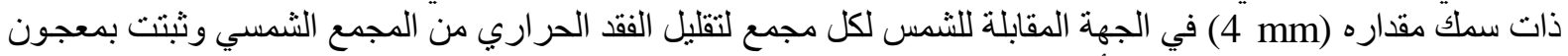

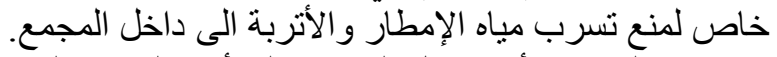

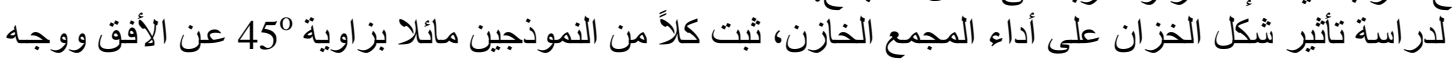

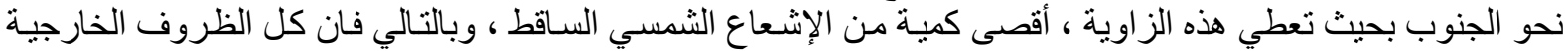

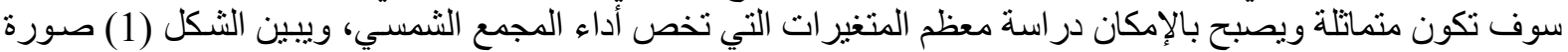
فوتو غر افية للمجمع الثمسي الخازن الاسطو اني و المكعب .

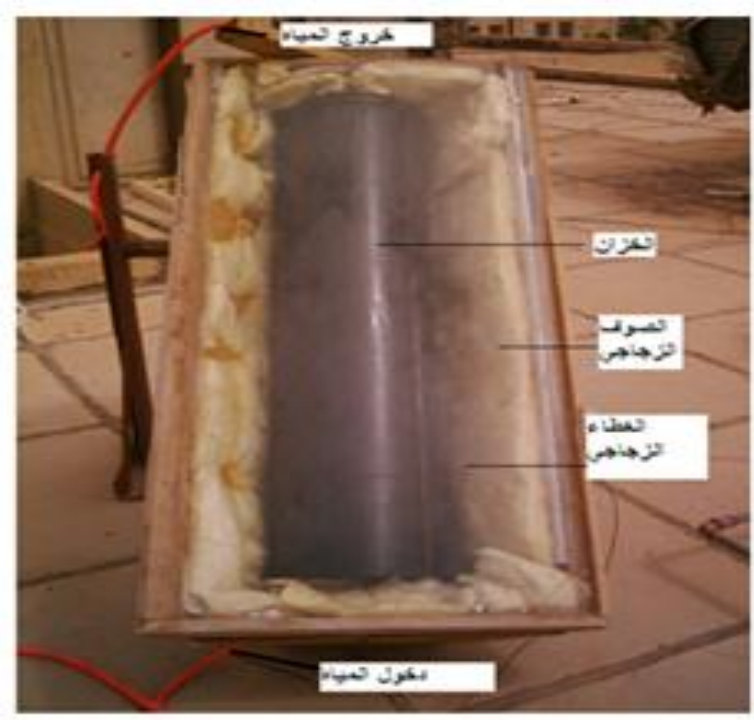

(أ)

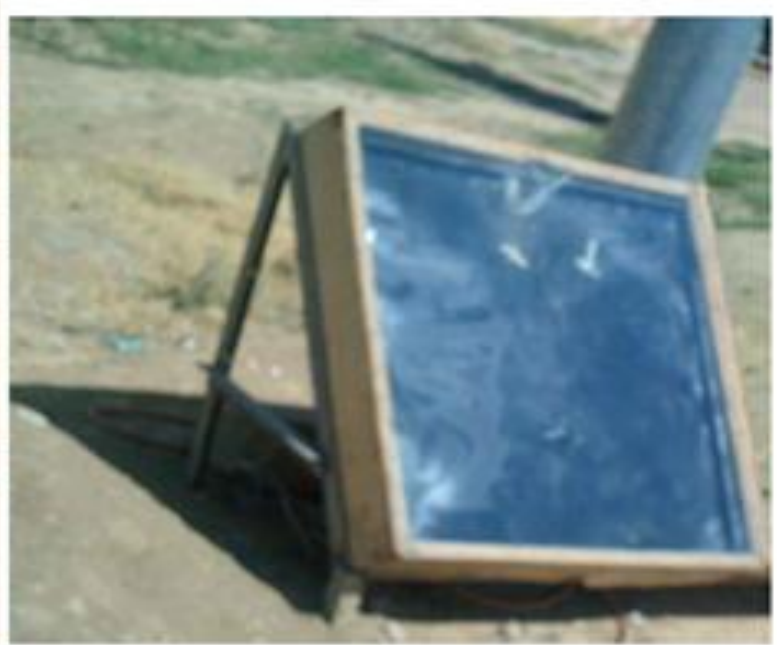

(ب)

الثكل (1) المنشأ التجريبي للبحث (أ. المجمع الاسطواني - ب.المجمع المكب)

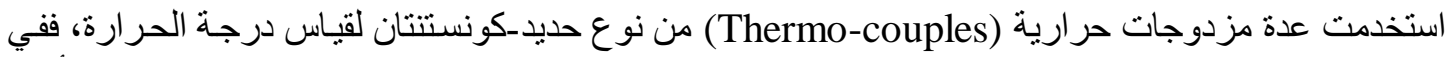

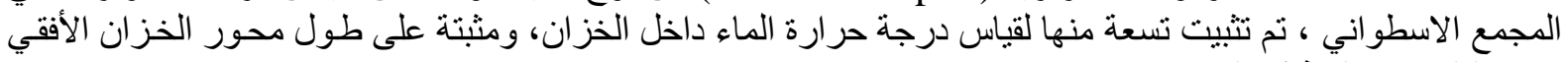
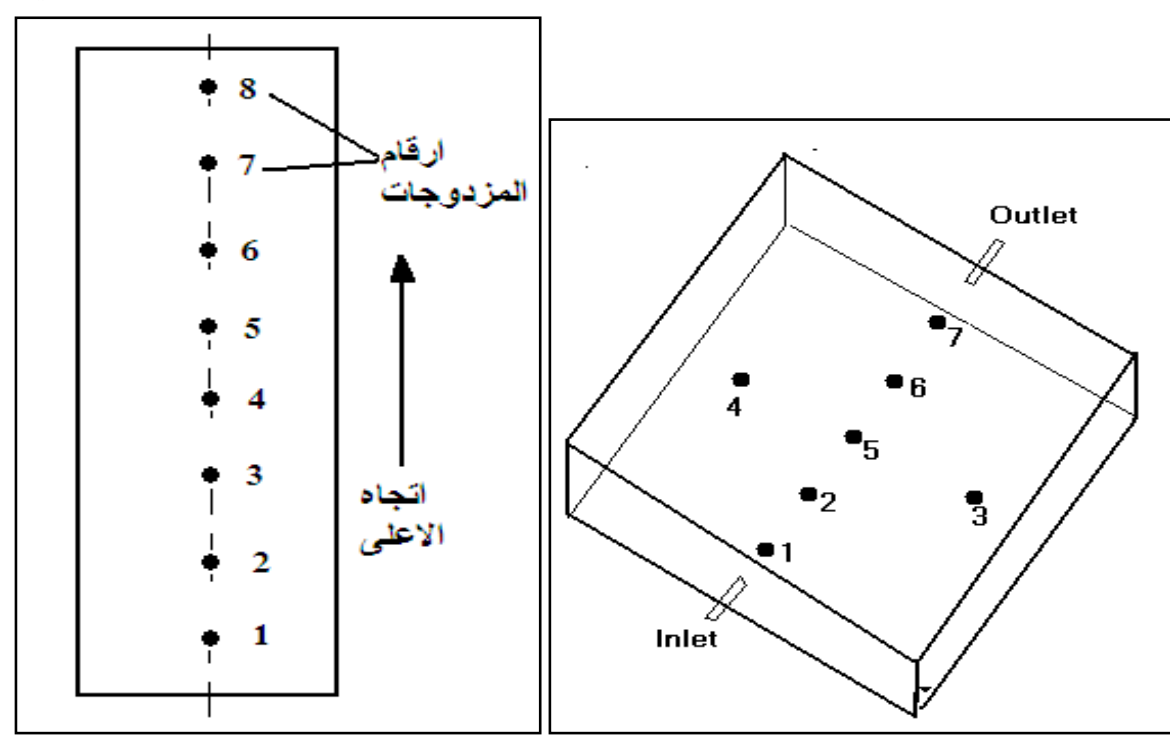

$$
\text { أـ المكب الشكل (2) مواقع المزدوجات الحرارية }
$$

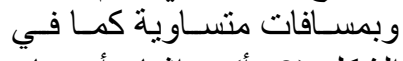

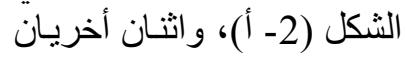

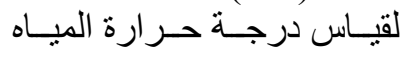

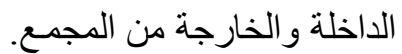

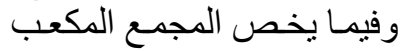

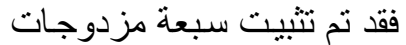

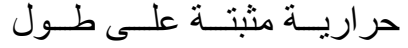

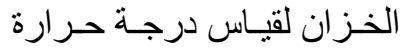
المباه داخل الخز ان وكما مبين في الثكل (2-ب)، إضافة إلى دئ

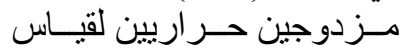

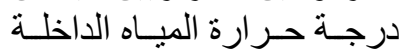

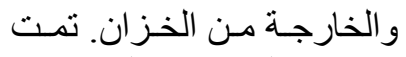

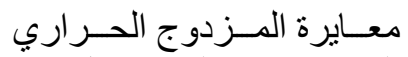

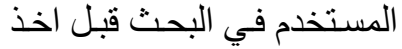
قر اءات درجات الحرارة نسبة فئة

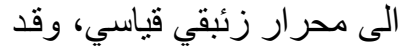
مثلت هذه القراءات في منحنى 


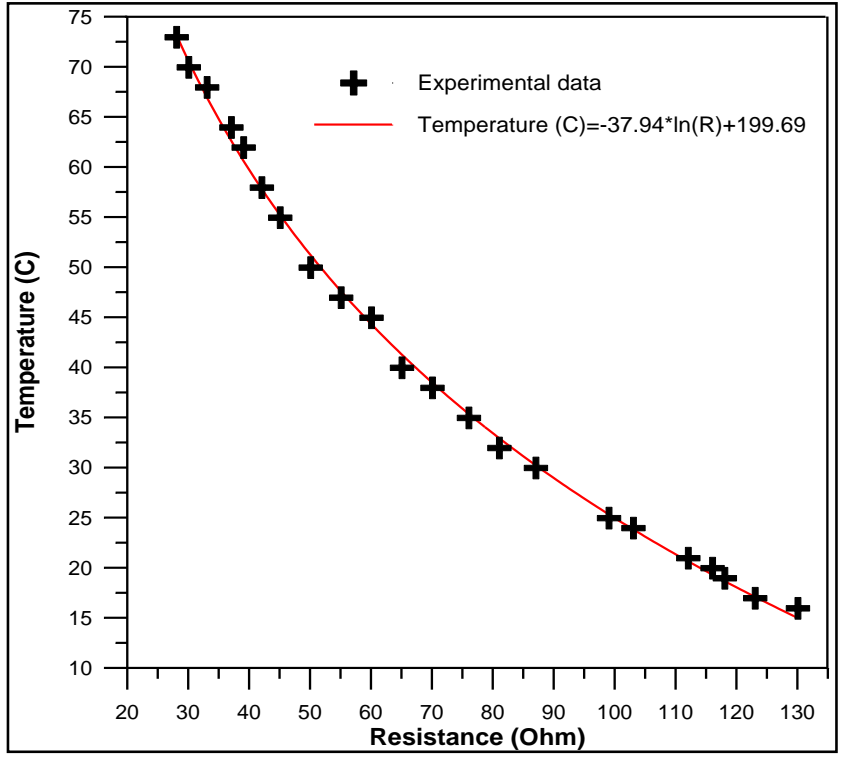

الشكل (3) منحنح، المعايرة للمزدو ج) الحرارى المستخدم

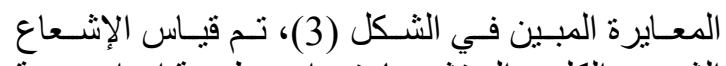

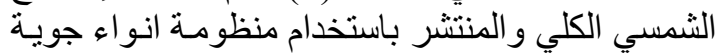

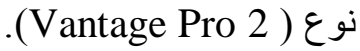

أجريت سلسلة تجارب منتظمة لدر اسـة الأداء الأمنل للمجمعين الشمسيين في البيئة العر اقية وبظروف لئنس

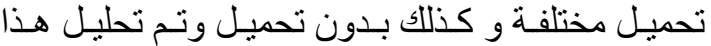

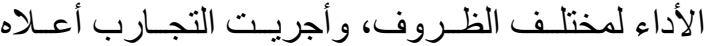

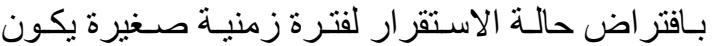
خلالها الإشعاع الشمسي وسر عة الرياح ودرجة الإنة حرارة

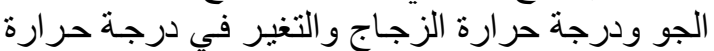

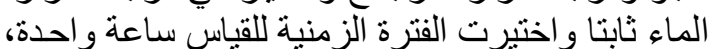
سجلت خلالها درجات الحرارة داخل الخز النات.

\section{3. الجانب النظري:}

اسـتخدم البرنـامج العددي (Fluent) لإنجـاز

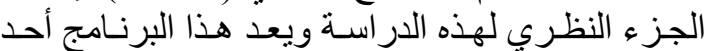

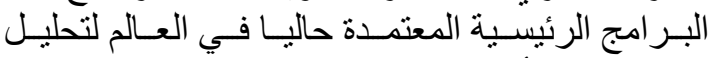

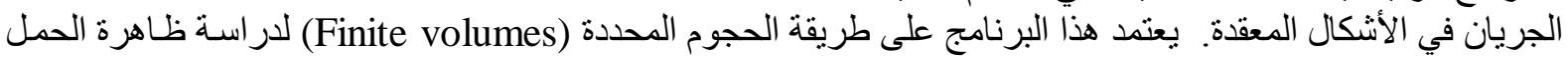

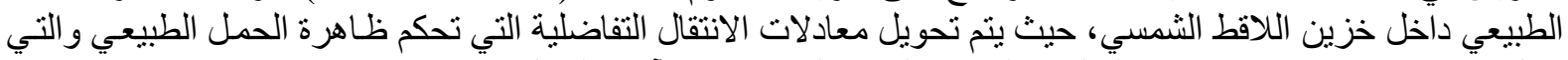
تمثل معادلات الاستمر ارية (حفظ الكتلة) و الزخم حون الطاقة إلى معادلات آنية بالثكل الآتي: $\frac{\partial(\boldsymbol{\rho} \phi)}{\partial t}+\frac{\partial(\boldsymbol{\rho} \boldsymbol{\phi})}{\partial \boldsymbol{x}}+\frac{\partial(\boldsymbol{\rho} \boldsymbol{\phi})}{\partial y}+\frac{\partial(\rho w \phi)}{\partial z}=\frac{\partial}{\partial x}\left(\boldsymbol{\Gamma} \frac{\partial \phi}{\partial x}\right)+$

$\frac{\partial}{\partial y}\left(\Gamma \frac{\partial \phi}{\partial y}\right)+\frac{\partial}{\partial z}\left(\Gamma \frac{\partial \phi}{\partial z}\right)+S$

حيث ان ض أي خاصية مثل السرعة ودرجة الحرارة و م معامل الانتشار، تحول المعادلة أعلاه باستخدام طريقة الحجوم المحددة إلى معادلات آنية يمكن بو اسطتها إيجاد قيمة أي خاصية في النقطة (P) مركز الحجم المسيطر (الثكل (4)) بدلالتة قيم الخاصية في النقاط المجاورة بالإن الثكل الآتي:

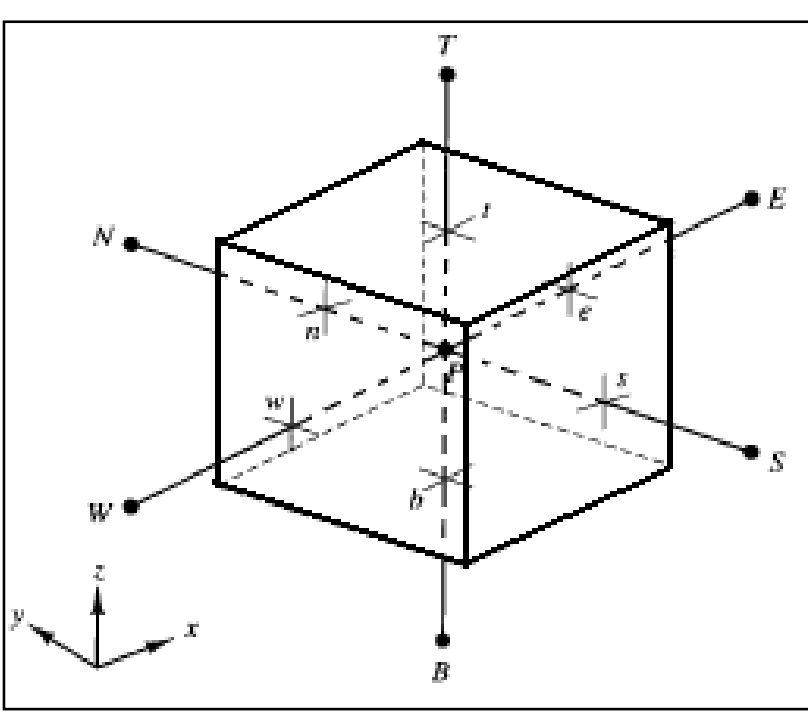

الثكل (4) الحجم المسيطر لطريقة الحجوم المحدة
$a_{P} \phi_{P}=a_{W} \phi_{W}+a_{E} \phi_{E}+a_{S} \phi_{S}+$

$a_{N} \phi_{N}+a_{B} \phi_{B}+a_{T} \phi_{T}+a_{P}^{0} \phi_{P}^{0}+S_{u}$

يتم تكامل كل معادلة من معادلات الاستمر ارية و الزخم

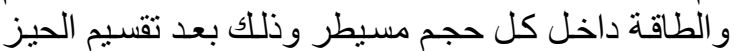

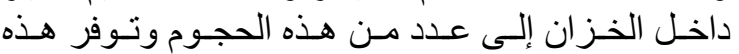

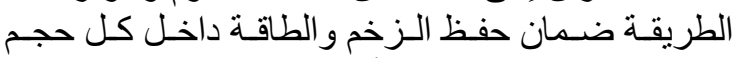
وبالتالي تعتبر هذه الطريقة أكثر قبو لا من طريقة الفروق الفرة دافي المحددة أو العناصر المنره المحدة.

اسـتخدم برنــامج ملحـق يســى (Gambit)

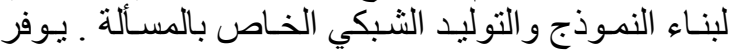

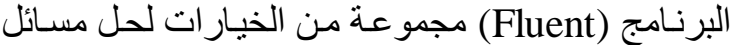

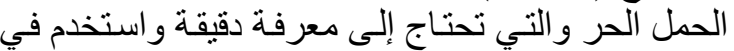

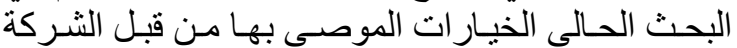

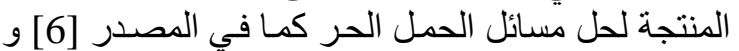

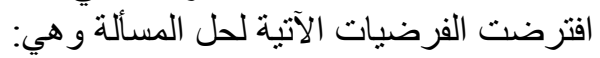
1. الحمل الطبيعي في الحيز طباقي ومعتمد على الزمن. لأن. 
2. جميع خواص الماء ثابتة عدا الكثافة وسوف يصار إلى اعتماد افتراض بوسنسك و الذي ينص:

$$
\rho \cong \rho_{o} *\left(1+\beta *\left(T_{o}-T\right)\right)
$$

3. اختيار الخوارزمية (PISO) لربط معادلات الزخم و الطاقة وهي ملائمة للحالات المعتمدة على الزمن.

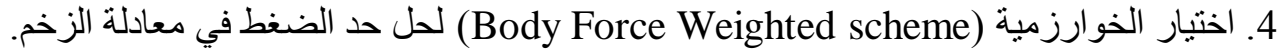

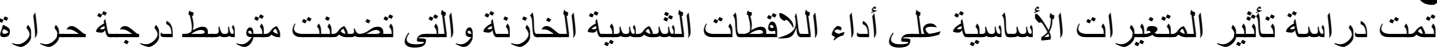
الخزين و توزيع درجات الحرارة وتأثير الحمل و أفضل مكان لإنخال الماء الجديد و سحب الماء الساء الساخن.

:Mean storage temperature

1.4 متوسط درجة حرارة الخزين

يعد متوسط درجة حرارة الخزين الخزين من أهم العو امل التي تحدد أداء السخان الثمسي الخـازن ويحسب هذا العامل

من المعادلة الآتية[7]:

$T_{a v}=\frac{\sum_{i=1}^{n} m_{i} * T_{i}}{m_{\text {total }}}$

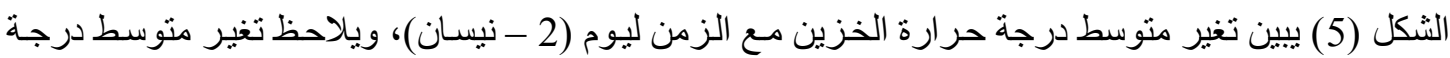

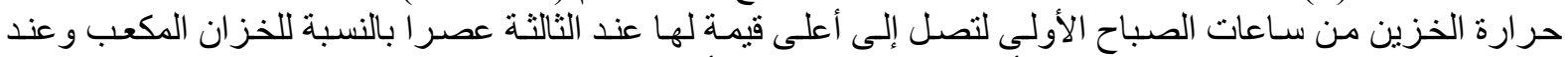

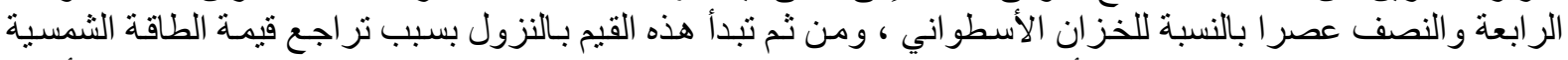

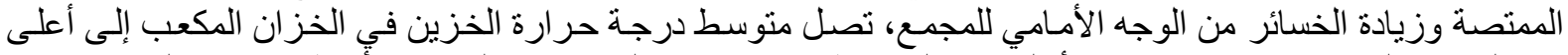

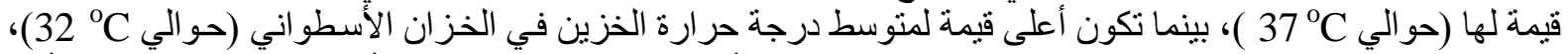

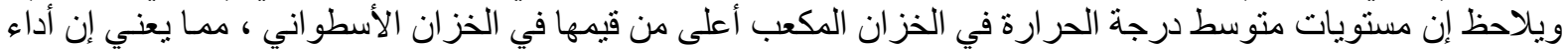

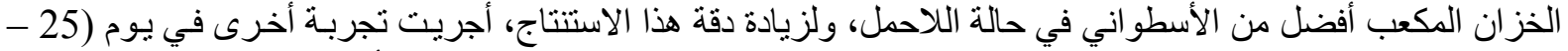

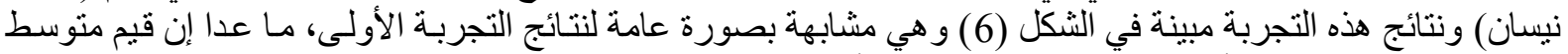

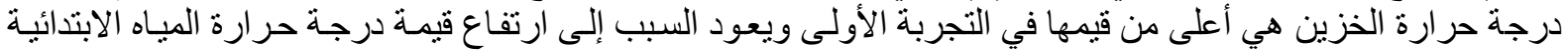

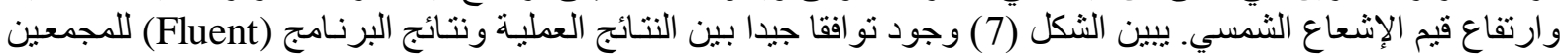

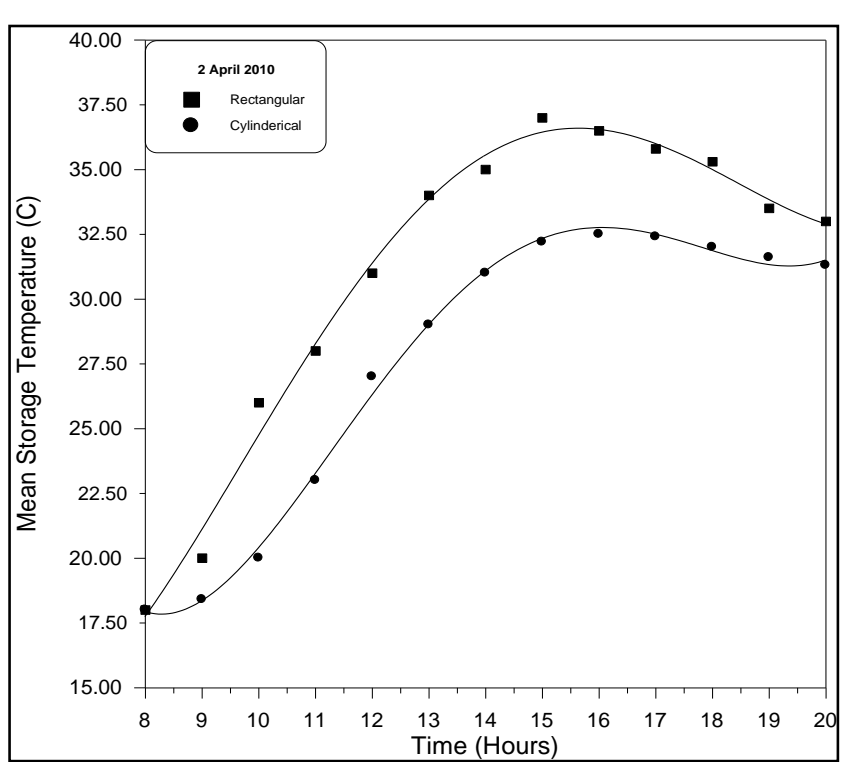

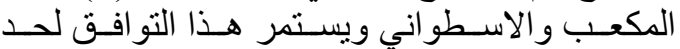

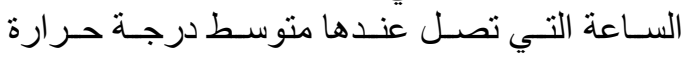

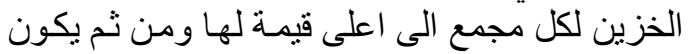

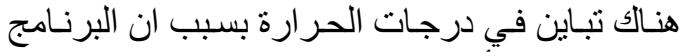

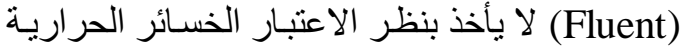

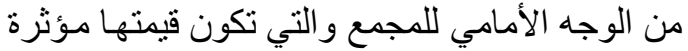

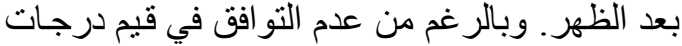

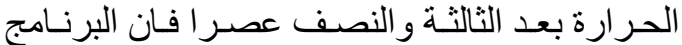
يعطي موثوقية جيدة يمكن الاعتماد عليها اليها

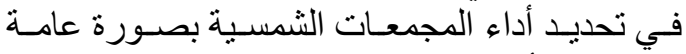
وبنسبة خطأ لا تتجاوز 4\% ـ

الشكل (5) تغير متوسط درجة حرارة الخزين مع الزمن ليوم 2-نيسان جران 


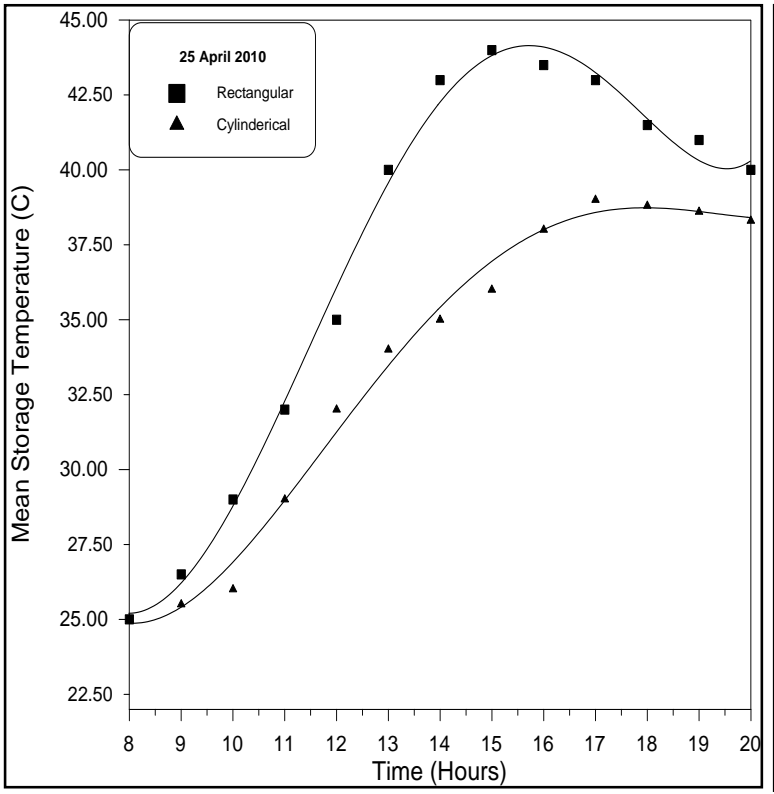

الشكل (7) تغير متوسط درجة حرارة الخزين نظريا وعمليا مع الزمن ليوم 2-نيسان

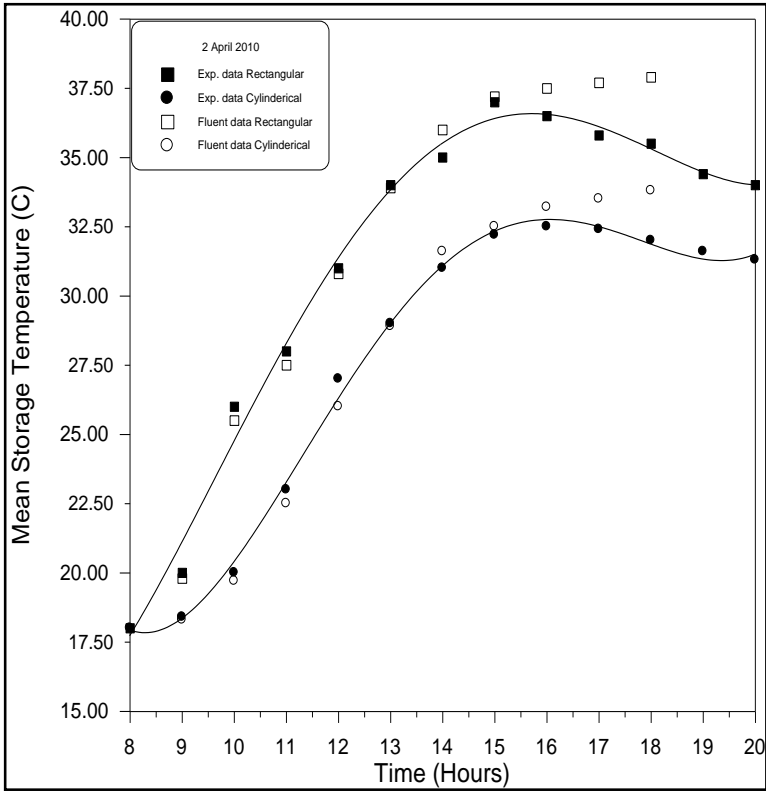

ألثكل (6) تغير متوسط درجة حرارة الخزين مع إنوم الزمن ليوم 25-نيسان

:Temperature distribution inside collector

2.4

يبين الثكل (8) التوزيع الحراري داخل المجمعات الثمسية عند منتصف النهار ( السـاعة الثانية عشر ) ولهذا

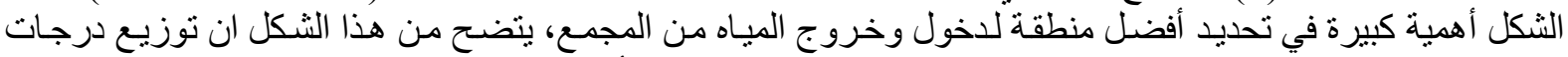

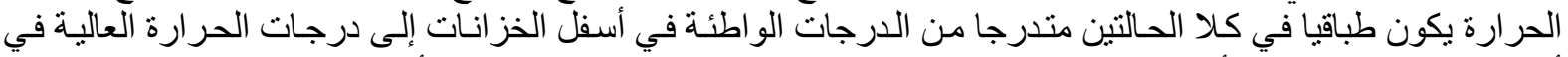

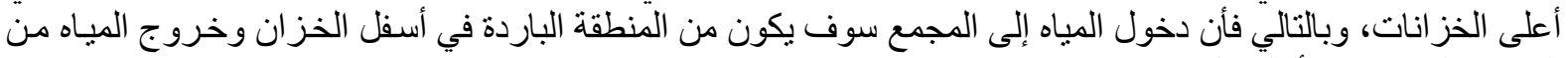
المنطقة الساخنة في أعلى المجمع.
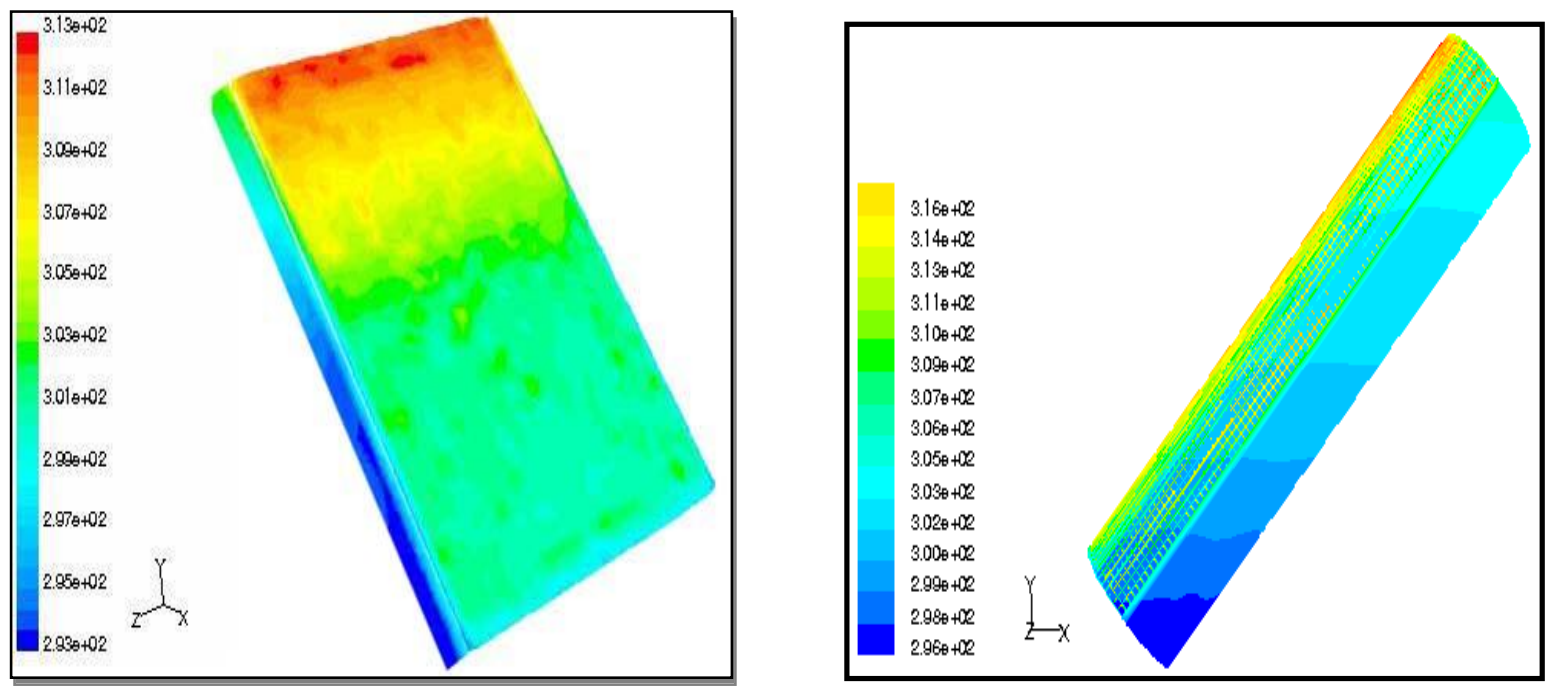

(ب) المجمع الاسطواني

الثكل (8) التوزيع الحراري داخل المجمعات الثمسية

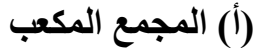




\section{:Effect of water withdrawal}

تختلف كمية المياه المصروفة من شخص ما على مجمو عة من العو امل، من اهمها المستوى الثقافي و الاجتماعي

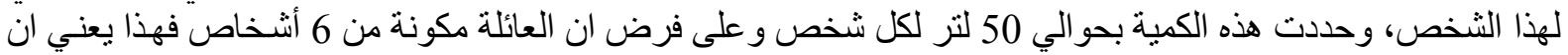

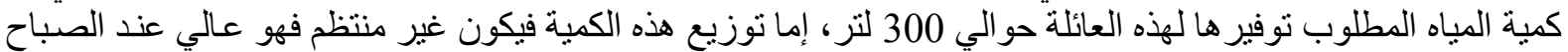

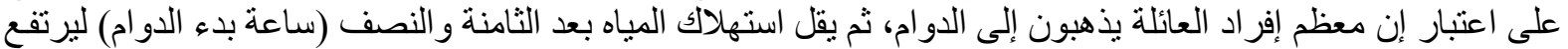

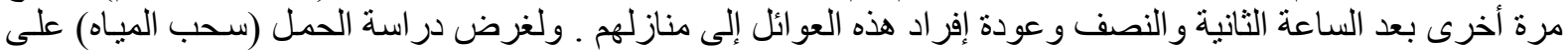
أداء هذه المجمعات، أجريت مجمو عة من التجارب لبياء لبيان هذا التأثير وفق التئ التوصيف الآتي:

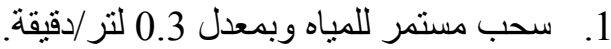
2.

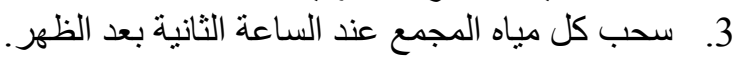

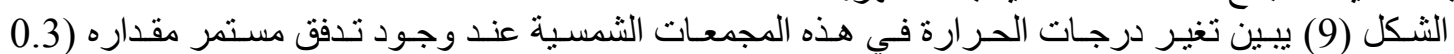

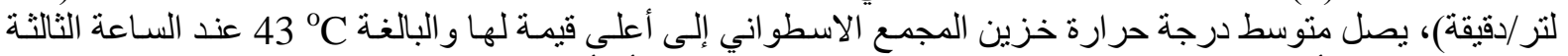

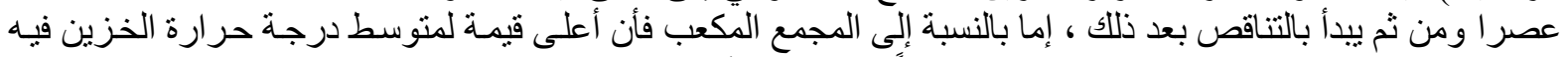

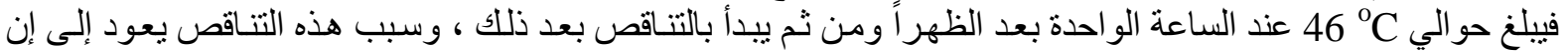

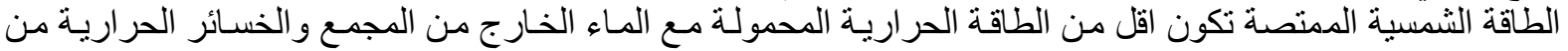

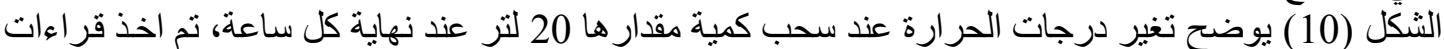

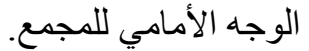

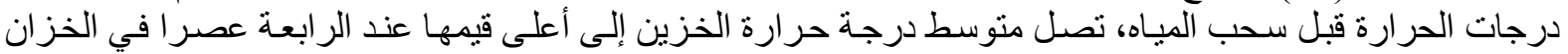

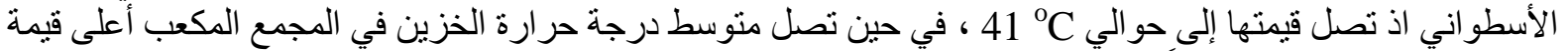

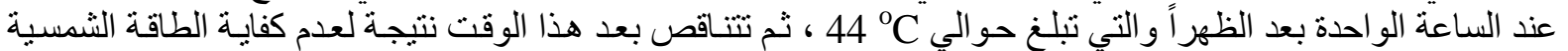

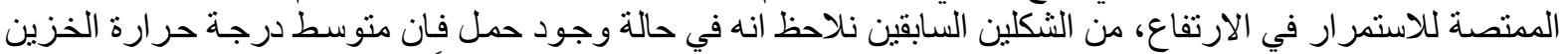

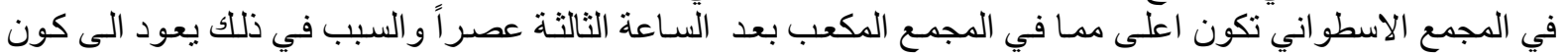

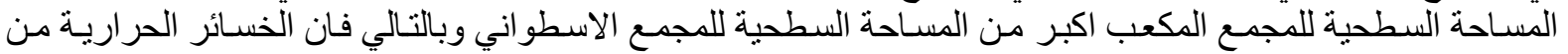

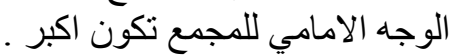

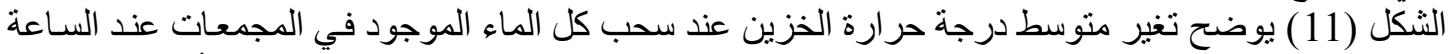

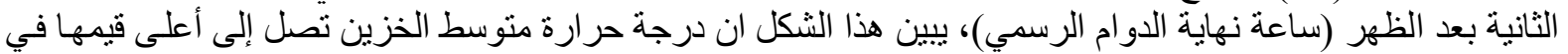

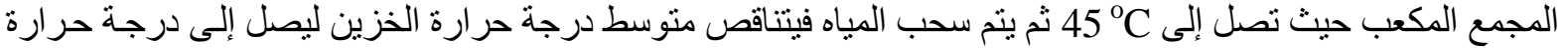

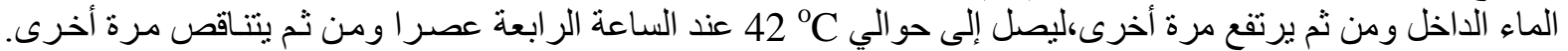

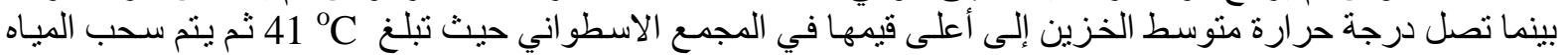

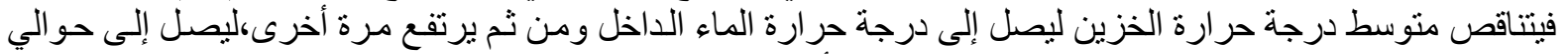

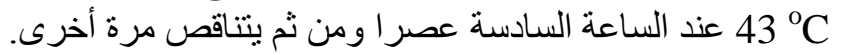

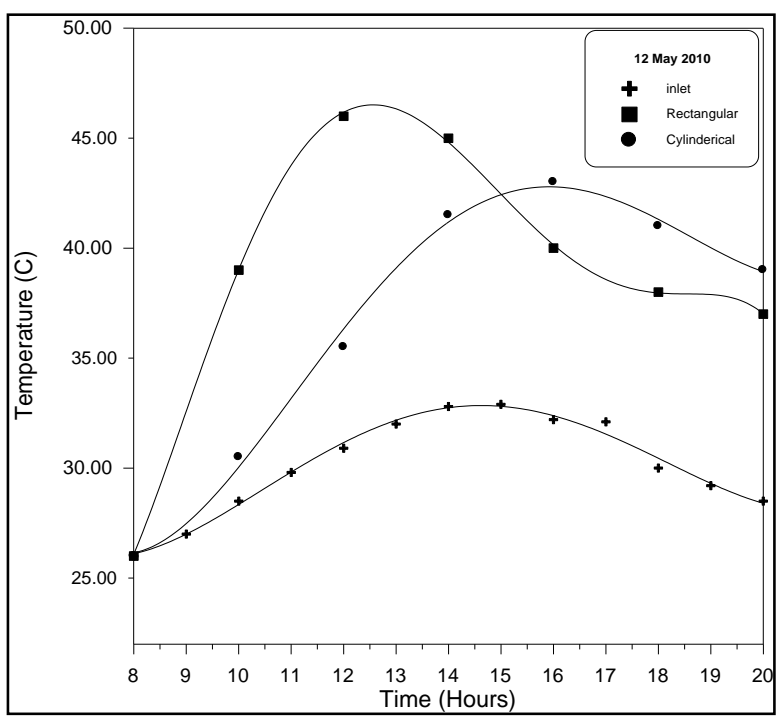

الثكل (9) تغير درجات الحرارة عند وجود تدفق

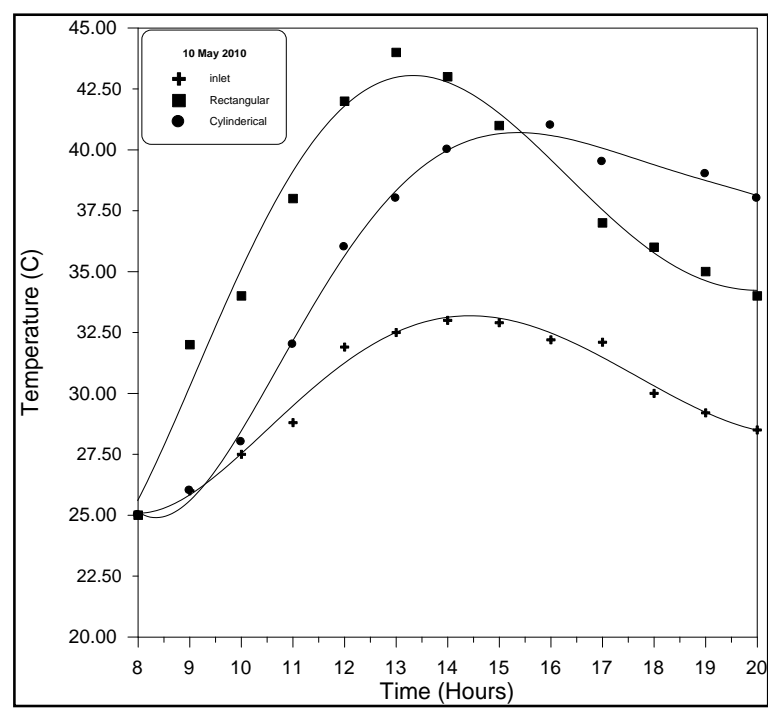

الثكل (10) تغير درجات الحرارة عند سحب كمية

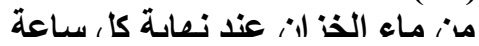




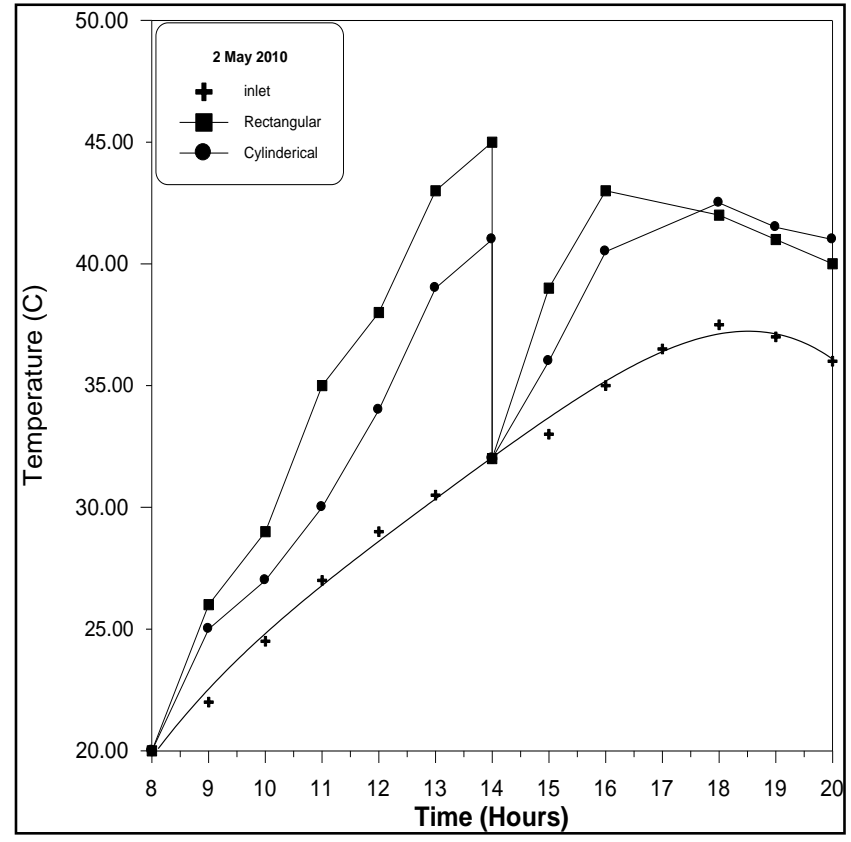

الثكل (11) تغير درجات الصرارة عند سحب ماء الخزان بصورة كاملة عند الثانية بعد الظهر عند مار

\section{5. الاستنتاجات والتوصيات:}

من خلال النتائج المستحصلة نجد ما بليلي:

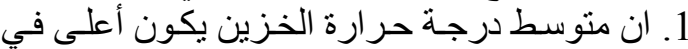

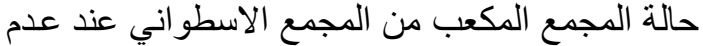

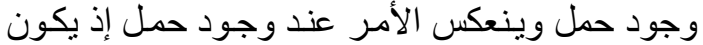

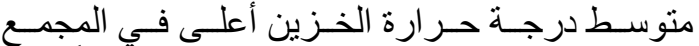

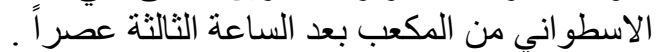

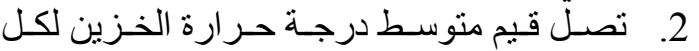
مجمع إلى أعلى قيمها عند ساعة معينة من النهار

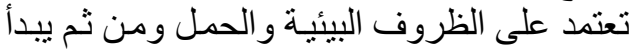

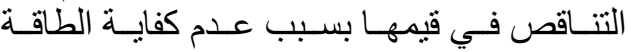
الشمسية الممتصة.

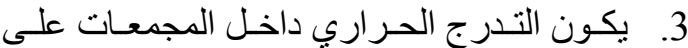
شكل طبقات، فالميـاه البـاردة في الأسفل و الميـاه

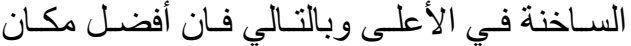

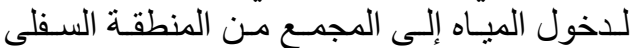

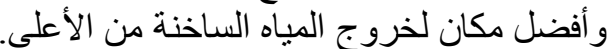

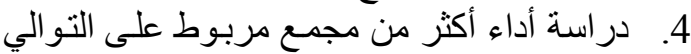

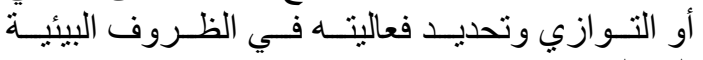
المختلفة 5. دراسة استخدام أكثر من غطاء زجـاجي لتحسين أداء هذه المجمعات الشمسية.

1. خضير مرزوق،1987، تجميع وخزن الطاقة الثمسية باستخدام الطبقة الحصوية ، رسالة ماجستير، كلية الهندسة، جامعة البصرة.

2. Duffie, J. and Beckman, W., 1980. Solar energy thermal processes, John Wiley and Sons, New York.

3. Khanna, M.L. ,1973, A portable-type solar water heater ,Solar energy, 15,pp 269-272.

4. Garge, H.P and Rani, U.; 1982. Theoretical and experimental studies on collector/storage type solar water heater, Solar energy, Vol.29, pp.467-478.

5. Farhan, .A; 2002. Computational model for a prism shaped storage solar collector with a right triangular cross section, M.Sc thesis, Mechanical engineering department, University of Baghdad.

6. Fluent 5.3 document, 1998,Fluent Inc.

7. Khalifa, A-J. N.; 1999. On the verification of one dimensional heat flow in a horizontal thermosyphon storage tank, J. of Energy Conversion and Management, Vol. 40, p. 961.

تم اجراء البحث في المعه التقني/الحويجة 